\title{
Approximation on the Quadratic Reciprocal Functional Equation
}

\author{
Abasalt Bodaghi ${ }^{1}$ and Sang Og Kim ${ }^{2}$ \\ ${ }^{1}$ Department of Mathematics, Garmsar Branch, Islamic Azad University, Garmsar, Iran \\ ${ }^{2}$ Department of Mathematics, Hallym University, Chuncheon 200-702, Republic of Korea \\ Correspondence should be addressed to Abasalt Bodaghi; abasalt.bodaghi@gmail.com
}

Received 19 November 2013; Accepted 9 January 2014; Published 20 February 2014

Academic Editor: Bing Xu

Copyright (C) 2014 A. Bodaghi and S. O. Kim. This is an open access article distributed under the Creative Commons Attribution License, which permits unrestricted use, distribution, and reproduction in any medium, provided the original work is properly cited.

The quadratic reciprocal functional equation is introduced. The Ulam stability problem for an $\epsilon$-quadratic reciprocal mapping $f: X \rightarrow Y$ between nonzero real numbers is solved. The Găvruţa stability for the quadratic reciprocal functional equations is established as well.

\section{Introduction}

In [1], Ulam proposed the well-known Ulam stability problem and one year later, the problem for linear mappings was solved by Hyers [2]. Bourgin [3] also studied the Ulam problem for additive mappings. Gruber [4] claimed that this kind of stability problem is of particular interest in the probability theory and in the case of functional equations of different types. The result of Hyers was generalized for approximately additive mappings by Aoki [5] and for approximately linear mappings, by considering the unbounded Cauchy differences by Rassias [6]. A further generalization was obtained by Găvruţa [7] by replacing the Cauchy differences with a control function $\varphi$ satisfying a very simple condition of convergence. Skof [8] was the first author to solve the Ulam problem for quadratic mappings on Banach algebras. Cholewa [9] demonstrated that the theorem of Skof is still true if relevant domain is replaced with an abelian group (see also [10-14]).

Ravi and Senthil Kumar [15] studied the Hyers-Ulam stability for the reciprocal functional equation

$$
f(x+y)=\frac{f(x) f(y)}{f(x)+f(y)},
$$

where $f: X \rightarrow Y$ is a mapping in the space of nonzero real numbers. It is easy to check that the reciprocal function $f(x)=$ $1 / x$ is a solution of the functional equation (1). Other results regarding the stability of various forms of the reciprocal functional equation can be found in [16-22].

In this paper, we study the Ulam-Găvruţa-Rassias stability for a new 2-dimensional quadratic reciprocal functional mapping $f: X \rightarrow Y$ satisfying the Rassias quadratic reciprocal functional equation

$$
f(2 x+y)+f(2 x-y)=\frac{2 f(x) f(y)[4 f(y)+f(x)]}{(4 f(y)-f(x))^{2}} .
$$

It is easily verified that the quadratic reciprocal function $f(x)=1 / x^{2}$ is a solution of the functional equation (2). As some corollaries, we investigate the pertinent stability of the Rassias equation (2) controlled by the "sum, product, and the mixed product-sum of powers of norms."

\section{2. $\epsilon$-Stability of $(2)$}

Throughout this paper, we denote the space of nonzero real numbers by $\mathbb{R}^{*}$.

Definition 1. A mapping $f: \mathbb{R}^{*} \rightarrow \mathbb{R}^{*}$ is called Rassias quadratic reciprocal, if the Rassias quadratic reciprocal functional equation (2) holds for all $x, y \in \mathbb{R}^{*}$.

Discussion on the above Definition and (2). We firstly note that, in the above definition, the equalities $x=y / 2$ and 
$x=-y / 2$ can not occur because $2 x-y$ and $2 x+y$ do not belong to $\mathbb{R}^{*}$. On the other hand, if $4 f(y)=f(x)$, we consider (2) which is equivalent to

$$
\begin{gathered}
(4 f(y)-f(x))^{2}(f(2 x+y)+f(2 x-y)) \\
=2 f(x) f(y)[4 f(y)+f(x)] .
\end{gathered}
$$

Since $f(y) \neq 0$, we have $f(x) \neq 0$. If $4 f(y)+f(x) \neq 0$, then $f(2 x+y)+f(2 x-y)$ is not defined. This is a contradiction. So, $4 f(y)+f(x)=0$. Hence, it follows that $f(x)=f(y)=$ $4 f(y)+f(x)=0$. However, in the case $4 f(y)+f(x)=0$, there is no problem in the definition of (2).

In the following theorem, we obtain an approximation for approximate quadratic reciprocal mappings on nonzero real numbers.

Theorem 2. Let $f: \mathbb{R}^{*} \rightarrow \mathbb{R}^{*}$ be a mapping for which there exists a constant $\epsilon$ (independent of $x$ and $y$ ) such that the functional inequality

$$
\begin{aligned}
& \left|f(2 x+y)+f(2 x-y)-\frac{2 f(x) f(y)[4 f(y)+f(x)]}{(4 f(y)-f(x))^{2}}\right| \\
& \quad \leq \frac{8}{9} \epsilon
\end{aligned}
$$

holds for all $x, y \in \mathbb{R}^{*}$. Then the limit

$$
Q(x)=\lim _{n \rightarrow \infty} \frac{1}{3^{2 n}} f\left(\frac{x}{3^{n}}\right)
$$

exists for all $x \in \mathbb{R}^{*}, n \in \mathbb{N}$ and $Q: \mathbb{R}^{*} \rightarrow \mathbb{R}^{*}$ is the unique mapping satisfying the Rassias quadratic reciprocal functional equation (2), such that

$$
|f(x)-Q(x)| \leq \epsilon
$$

for all $x \in \mathbb{R}^{*}$. Moreover, the functional identity

$$
Q(x)=\frac{1}{3^{2 n}} Q\left(\frac{x}{3^{n}}\right)
$$

holds for all $x \in \mathbb{R}^{*}$ and $n \in \mathbb{N}$.

Proof. Putting $y=x$ in (4), we get

$$
\left|f(3 x)-\frac{1}{3^{2}} f(x)\right| \leq \frac{8 \epsilon}{9}
$$

for all $x \in \mathbb{R}^{*}$. Thus we have

$$
\left|f(x)-\frac{1}{3^{2}} f\left(\frac{x}{3}\right)\right| \leq \frac{8 \epsilon}{9}
$$

for all $x \in \mathbb{R}^{*}$. Substituting $x$ by $x / 3$ in (9) and then dividing both sides by $3^{2}$, we obtain

$$
\left|\frac{1}{3^{2}} f\left(\frac{x}{3}\right)-\frac{1}{3^{4}} f\left(\frac{x}{3^{2}}\right)\right| \leq \frac{8 \epsilon}{3^{4}}
$$

for all $x \in \mathbb{R}^{*}$. It follows from (9) and (10) that

$$
\left|f(x)-\frac{1}{3^{4}} f\left(\frac{x}{3^{2}}\right)\right| \leq \frac{8 \epsilon}{9}\left(1+\frac{1}{3^{2}}\right)
$$

for all $x \in \mathbb{R}^{*}$. The above process can be repeated to obtain

$$
\left|f(x)-\frac{1}{3^{2 n}} f\left(\frac{x}{3^{n}}\right)\right| \leq \frac{8 \epsilon}{9}\left(1+\frac{1}{3^{2}}+\frac{1}{3^{4}}+\cdots+\frac{1}{3^{2(n-1)}}\right)
$$

for all $x \in \mathbb{R}^{*}$ and all $n \in \mathbb{N}$. In order to prove the convergence of the sequence $\left\{\left(1 / 3^{2 n}\right) f\left(x / 3^{n}\right)\right\}$, we have if $n>k>0$, then by (12)

$$
\begin{aligned}
\mid \frac{1}{3^{2 n}} & f\left(\frac{x}{3^{n}}\right)-\frac{1}{3^{2 k}} f\left(\frac{x}{3^{k}}\right) \mid \\
& =\frac{1}{3^{2 k}}\left|\frac{1}{3^{2(n-k)}}\left(\frac{x}{3^{n}}\right)-f\left(\frac{x}{3^{k}}\right)\right| \\
& =\frac{1}{3^{2 k}}\left|\frac{1}{3^{2(n-k)}} f\left(\frac{y}{3^{(n-k)}}\right)-f(y)\right| \\
& \leq \frac{\epsilon}{3^{2 k}} \frac{8}{9}\left(1+\frac{1}{3^{2}}+\frac{1}{3^{4}}+\cdots+\frac{1}{3^{2(n-k-1)}}\right) \\
& =\left(3^{-2 k}-3^{-2 n}\right) \epsilon \\
& \leq 3^{-2 k} \epsilon
\end{aligned}
$$

for all $x \in \mathbb{R}^{*}$ in which $y=x / 3^{k}$. The above relation shows that the mentioned sequence is a Cauchy sequence and thus limit (5) exists for all $x \in \mathbb{R}^{*}$. Taking that $n$ tends to infinity in (12), we can see that inequality (6) holds for all $x \in \mathbb{R}^{*}$. Replacing $x, y$ by $x / 3^{n}, y / 3^{n}$, respectively, in (4) and dividing both sides by $3^{2 n}$, we deduce that

$$
\begin{aligned}
\frac{1}{3^{2 n}} \mid & f\left(\frac{2 x+y}{3^{n}}\right)+f\left(\frac{2 x-y}{3^{n}}\right) \\
& -\frac{2 f\left(x / 3^{n}\right) f\left(y / 3^{n}\right)\left[4 f\left(y / 3^{n}\right)+f\left(x / 3^{n}\right)\right]}{\left(4 f\left(y / 3^{n}\right)-f\left(x / 3^{n}\right)\right)^{2}} \mid \\
& \leq \frac{8 \epsilon}{3^{2(n+1)}}
\end{aligned}
$$

holds for all $x, y \in \mathbb{R}^{*}$. Allowing $n \rightarrow \infty$ in (14), we see that $Q$ satisfies (2) for all $x, y \in \mathbb{R}^{*}$. To prove that $Q$ is a unique quadratic reciprocal function satisfying (2) subject to (6), let us consider a $Q: \mathbb{R}^{*} \rightarrow \mathbb{R}^{*}$ to be another quadratic reciprocal function which satisfies (2) and inequality (6). Clearly $Q$ and $Q$ satisfy (7) and using (6), we get

$$
\begin{aligned}
|Q(x)-\mathscr{Q}(x)|= & \lim _{n \rightarrow \infty} \frac{1}{3^{2 n}}\left|Q\left(\frac{x}{3^{n}}\right)-Q\left(\frac{x}{3^{n}}\right)\right| \\
\leq \lim _{n \rightarrow \infty} \frac{1}{3^{2 n}} & {\left[\left|Q\left(\frac{x}{3^{n}}\right)-f\left(\frac{x}{3^{n}}\right)\right|\right.} \\
& \left.+\left|f\left(\frac{x}{3^{n}}\right)-Q\left(\frac{x}{3^{n}}\right)\right|\right] \\
\leq & \lim _{n \rightarrow \infty} \frac{2 \epsilon}{3^{2 n}}=0,
\end{aligned}
$$

for all $x \in \mathbb{R}^{*}$. This shows the uniqueness of $Q$. 


\section{Găvruţa Stability of (2)}

Theorem 3. Let $l \in\{1,-1\}$ be fixed. Suppose that $F: \mathbb{R}^{*} \times$ $\mathbb{R}^{*} \rightarrow \mathbb{R}^{*}$ is a function such that

$$
\sum_{n=1}^{\infty} \frac{1}{3^{2 l(n-l)}} F\left(\frac{x}{3^{\ln }}, \frac{x}{3^{\ln }}\right)<\infty
$$

for all $x \in \mathbb{R}^{*}$. Assume in addition that $f: \mathbb{R}^{*} \rightarrow \mathbb{R}^{*}$ is a function which satisfies the functional inequality

$$
\begin{aligned}
& \left|f(2 x+y)+f(2 x-y)-\frac{2 f(x) f(y)[4 f(y)+f(x)]}{(4 f(y)-f(x))^{2}}\right| \\
& \quad \leq F(x, y)
\end{aligned}
$$

holds for all $x, y \in \mathbb{R}^{*}$. Then there exists a unique quadratic reciprocal function $Q: \mathbb{R}^{*} \rightarrow \mathbb{R}^{*}$ which satisfies the Rassias equation (2) and the inequality

$$
|f(x)-Q(x)| \leq \sum_{n=|l+1| / 2}^{\infty} \frac{1}{3^{2 l(n-l)}} F\left(\frac{x}{3^{\ln }}, \frac{x}{3^{\ln }}\right)
$$

for all $x \in \mathbb{R}^{*}$.

Proof. We prove the result only in the case that $l=1$. Another case is similar. Putting $y=x$ in (17), we have

$$
\left|f(3 x)-\frac{1}{3^{2}} f(x)\right| \leq F(x, x)
$$

for all $x \in \mathbb{R}^{*}$. Replacing $x$ by $x / 3$ in the above inequality, we get

$$
\left|f(x)-\frac{1}{3^{2}} f\left(\frac{x}{3}\right)\right| \leq F\left(\frac{x}{3}, \frac{x}{3}\right)
$$

for all $x \in \mathbb{R}^{*}$. Replacing $x$ by $x / 3^{n}$ in (20) and then dividing both sides by $3^{2 n}$, we have

$$
\left|\frac{1}{3^{2 n}} f\left(\frac{x}{3^{n}}\right)-\frac{1}{3^{2(n+1)}} f\left(\frac{x}{3^{n+1}}\right)\right| \leq \frac{1}{3^{2 n}} F\left(\frac{x}{3^{n}}, \frac{x}{3^{n}}\right)
$$

for all $x \in \mathbb{R}^{*}$ and all nonnegative integers $n$. Thus the sequence $\left\{\left(1 / 3^{2 n}\right) f\left(x / 3^{n}\right)\right\}$ is Cauchy by (16) and so this sequence is convergent. Indeed,

$$
\lim _{n \rightarrow \infty} \frac{1}{3^{2 n}} f\left(\frac{x}{3^{n}}\right)=Q(x)
$$

for all $x \in \mathbb{R}^{*}$. On the other hand, by using (20) and applying mathematical induction to a positive integer $n$, we obtain

$$
\left|f(x)-\frac{1}{3^{2 n}} f\left(\frac{x}{3^{n}}\right)\right| \leq \sum_{k=1}^{n} \frac{1}{3^{2(k-1)}} F\left(\frac{x}{3^{k}}, \frac{x}{3^{k}}\right)
$$

for all $x \in \mathbb{R}^{*}$ and all $n \in \mathbb{N}$. Letting $n \rightarrow \infty$ in (23) and using (22), one sees that inequality (18) holds for all $x \in \mathbb{R}^{*}$.
Replacing $x, y$ by $x / 3^{n}, y / 3^{n}$ in (17) and dividing both sides by $3^{2 n}$, we deduce that

$$
\begin{aligned}
\frac{1}{3^{2 n}} \mid & f\left(\frac{2 x+y}{3^{n}}\right)+f\left(\frac{2 x-y}{3^{n}}\right) \\
& -\frac{2 f\left(x / 3^{n}\right) f\left(y / 3^{n}\right)\left[4 f\left(y / 3^{n}\right)+f\left(x / 3^{n}\right)\right]}{\left(4 f\left(y / 3^{n}\right)-f\left(x / 3^{n}\right)\right)^{2}} \mid \\
& \leq \frac{1}{3^{2 n}} F\left(\frac{x}{3^{n}}, \frac{x}{3^{n}}\right)
\end{aligned}
$$

holds for all $x, y \in \mathbb{R}^{*}$. Taking $n \rightarrow \infty$ in (24), we see that $Q$ satisfies (2) for all $x, y \in \mathbb{R}^{*}$. Now, let $Q^{\prime}: \mathbb{R}^{*} \rightarrow \mathbb{R}^{*}$ be another quadratic reciprocal function which satisfies (2) and inequality (18). Obviously $Q$ and $Q^{\prime}$ satisfy (7). Using (18), we get

$$
\begin{aligned}
\left|Q(x)-Q^{\prime}(x)\right| & =\frac{1}{3^{2 n}}\left|Q\left(\frac{x}{3^{n}}\right)-Q^{\prime}\left(\frac{x}{3^{n}}\right)\right| \\
\leq & \frac{1}{3^{2 n}}\left[\left|Q\left(\frac{x}{3^{n}}\right)-f\left(\frac{x}{3^{n}}\right)\right|\right. \\
& \left.\quad+\left|f\left(\frac{x}{3^{n}}\right)-Q^{\prime}\left(\frac{x}{3^{n}}\right)\right|\right] \\
\leq & \frac{2}{3^{2 n}} \sum_{k=1}^{\infty} \frac{1}{3^{2(k-1)}} F\left(\frac{x}{3^{k+n}}, \frac{x}{3^{k+n}}\right) \\
& =\sum_{k=1}^{\infty} \frac{2}{3^{2(k+n-1)}} F\left(\frac{x}{3^{k+n}}, \frac{x}{3^{k+n}}\right) \\
& =\sum_{k=n}^{\infty} \frac{2}{3^{2(k-1)}} F\left(\frac{x}{3^{k}}, \frac{x}{3^{k}}\right)
\end{aligned}
$$

for all $x \in \mathbb{R}^{*}$. Taking $n \rightarrow \infty$ in the preceding inequality, we immediately find the uniqueness of $Q$. For $l=-1$, we obtain

$$
\left|f(x)-3^{2 n} f\left(3^{n} x\right)\right| \leq \sum_{k=0}^{n-1} 3^{2(k+1)} F\left(3^{k} x, 3^{k} x\right)
$$

from which one can prove the result by a similar technique. This completes the proof.

Corollary 4. Let $\alpha, r$ be nonnegative real numbers with $r \neq-2$. Suppose that $f: \mathbb{R}^{*} \rightarrow \mathbb{R}^{*}$ is a function which satisfies the functional inequality

$$
\begin{aligned}
& \left|f(2 x+y)+f(2 x-y)-\frac{2 f(x) f(y)[4 f(y)+f(x)]}{(4 f(y)-f(x))^{2}}\right| \\
& \leq \alpha\left(|x|^{r}+|y|^{r}\right)
\end{aligned}
$$

for all $x, y \in \mathbb{R}^{*}$. Then there exists a unique quadratic reciprocal function $Q: \mathbb{R}^{*} \rightarrow \mathbb{R}^{*}$ that satisfies the Rassias equation (2) and the inequality

$$
|f(x)-Q(x)| \leq \frac{18 \alpha}{\left|3^{r+2}-1\right|}|x|^{r}
$$

for all $x \in \mathbb{R}^{*}$. 
Proof. Letting $F(x, y)=\alpha\left(|x|^{r}+|y|^{r}\right)$ in Theorem 3, we can get the result.

Corollary 5. Let $\alpha, r, s$ be nonnegative real numbers such that $\rho=r+s \neq-2$. Suppose that $f: \mathbb{R}^{*} \rightarrow \mathbb{R}^{*}$ is a function which satisfies the functional inequality

$$
\begin{aligned}
& \left|f(2 x+y)+f(2 x-y)-\frac{2 f(x) f(y)[4 f(y)+f(x)]}{(4 f(y)-f(x))^{2}}\right| \\
& \quad \leq \alpha|x|^{r}|y|^{s}
\end{aligned}
$$

for all $x, y \in \mathbb{R}^{*}$. Then there exists a unique quadratic reciprocal function $Q: \mathbb{R}^{*} \rightarrow \mathbb{R}^{*}$ that satisfies the Rassias equation (2) and the inequality

$$
|f(x)-Q(x)| \leq \frac{9 \alpha}{\left|3^{\rho+2}-1\right|}|x|^{\rho}
$$

for all $x \in \mathbb{R}^{*}$.

Proof. Defining $F(x, y)=\alpha|x|^{r}|y|^{s}$ and applying Theorem 3, one can obtain the desired result.

The proof of the following corollary is similar to the above results, so it is omitted.

Corollary 6. Let $\alpha, r$ be nonnegative real numbers with $r \neq-1$. Suppose that $f: \mathbb{R}^{*} \rightarrow \mathbb{R}^{*}$ is a function which satisfies the functional inequality

$$
\begin{aligned}
& \left|f(2 x+y)+f(2 x-y)-\frac{2 f(x) f(y)[4 f(y)+f(x)]}{(4 f(y)-f(x))^{2}}\right| \\
& \leq \alpha\left(|x|^{r}|y|^{r}+|x|^{2 r}+|y|^{2 r}\right)
\end{aligned}
$$

for all $x, y \in \mathbb{R}^{*}$. Then there exists a unique quadratic reciprocal function $Q: \mathbb{R}^{*} \rightarrow \mathbb{R}^{*}$ that satisfies the Rassias equation (2) and the inequality

$$
|f(x)-Q(x)| \leq \frac{27 \alpha}{\left|3^{2 r+2}-1\right|}|x|^{2 r}
$$

for all $x \in \mathbb{R}^{*}$.

\section{Conflict of Interests}

The authors declare that there is no conflict of interests regarding the publication of this paper.

\section{Acknowledgment}

The authors sincerely thank the anonymous reviewers for their careful reading, constructive comments, and fruitful suggestions to improve the quality of the first draft.

\section{References}

[1] S. M. Ulam, Problems in Modern Mathematics, chapter 6, John Wiley \& Sons, New York, NY, USA, 1940.

[2] D. H. Hyers, "On the stability of the linear functional equation," Proceedings of the National Academy of Sciences of the United States of America, vol. 27, pp. 222-224, 1941.

[3] D. G. Bourgin, "Classes of transformations and bordering transformations," Bulletin of the American Mathematical Society, vol. 57, pp. 223-237, 1951.

[4] P. M. Gruber, "Stability of isometries," Transactions of the American Mathematical Society, vol. 245, pp. 263-277, 1978.

[5] T. Aoki, "On the stability of the linear transformation in Banach spaces," Journal of the Mathematical Society of Japan, vol. 2, pp. 64-66, 1950.

[6] T. M. Rassias, "On the stability of the linear mapping in Banach spaces," Proceedings of the American Mathematical Society, vol. 72, no. 2, pp. 297-300, 1978.

[7] P. Găvruța, "A generalization of the Hyers-Ulam-Rassias stability of approximately additive mappings," Journal of Mathematical Analysis and Applications, vol. 184, no. 3, pp. 431-436, 1994.

[8] F. Skof, "Local properties and approximation of operators," Rendiconti del Seminario Matematico e Fisico di Milano, vol. 53, pp. 113-129, 1983.

[9] P. W. Cholewa, "Remarks on the stability of functional equations," Aequationes Mathematicae, vol. 27, no. 1-2, pp. 76-86, 1984.

[10] A. Bodaghi and I. A. Alias, "Approximate ternary quadratic derivations on ternary Banach algebras and $C^{*}$-ternary rings," Advances in Difference Equations, vol. 2012, article 11, 2012.

[11] S. Czerwik, "On the stability of the quadratic mapping in normed spaces," Abhandlungen aus dem Mathematischen Seminar der Universität Hamburg, vol. 62, pp. 59-64, 1992.

[12] M. Eshaghi Gordji and A. Bodaghi, "On the Hyers-Ulam-Rassias stability problem for quadratic functional equations," East Journal on Approximations, vol. 16, no. 2, pp. 123-130, 2010.

[13] J. R. Lee, J. S. An, and C. Park, "On the stability of quadratic functional equations," Abstract and Applied Analysis, vol. 2008, Article ID 628178, 8 pages, 2008.

[14] T. M. Rassias, "On the stability of the quadratic functional equation and its applications," Studia Universitatis Babes-Bolyai, vol. 43, no. 3, pp. 89-124, 1998.

[15] K. Ravi and B. V. Senthil Kumar, "Ulam-Gavruta-Rassias stability of Rassias Reciprocal functional equation," Global Journal of Applied Mathematics and Mathematical Sciences, vol. 3, no. 1-2, pp. 57-79, 2010.

[16] K. Ravi, J. M. Rassias, and B. V. Senthil Kumar, "Generalized Hyers-Ulam stability and examples of non-stability of reciprocal type functional equation in several variables," Indian Journal of Science, vol. 2, no. 3, pp. 13-22, 2013.

[17] K. Ravi, J. M. Rassias, and B. V. Senthil Kumar, "Stability of reciprocal difference and adjoint functional equations in paranormed spaces: direct and fixed point methods," Functional Analysis, Approximation and Computation, vol. 5, no. 1, pp. 5772, 2013.

[18] K. Ravi, J. M. Rassias, and B. V. Senthil Kumar, "Example for the stability of reciprocal type functional equation controlled by product of different powers in singular case," Advances in Pure Mathematics, vol. 61, pp. 16821-16825, 2013.

[19] K. Ravi, J. M. Rassias, and B. V. Senthil Kumar, "Ulam stability of generalized reciprocal functional equation in several variables," 
International Journal of Applied Mathematics \& Statistics, vol. 19, no. D10, pp. 1-19, 2010.

[20] K. Ravi, J. M. Rassias, and B. V. Senthil Kumar, "A fixed point approach to the generalized Hyers-Ulam stability of reciprocal difference and adjoint functional equations," Thai Journal of Mathematics, vol. 8, no. 3, pp. 469-481, 2010.

[21] K. Ravi, J. M. Rassias, and B. V. Senthil Kumar, "Ulam stability of reciprocal difference and adjoint functional equations," The Australian Journal of Mathematical Analysis and Applications, vol. 8, no. 1, article 13, pp. 1-18, 2011.

[22] K. Ravi, E. Thandapani, and B. V. Senthil Kumar, "Stability of reciprocal type functional equations," Panamerican Mathematical Journal, vol. 21, no. 1, pp. 59-70, 2011. 


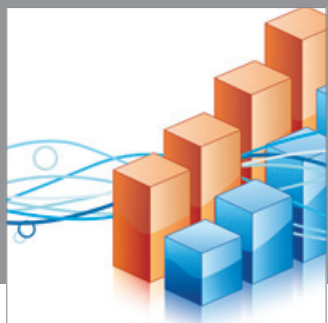

Advances in

Operations Research

mansans

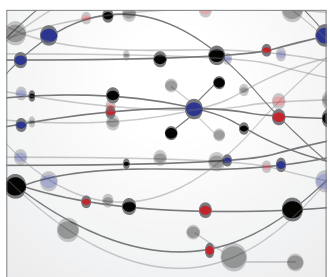

The Scientific World Journal
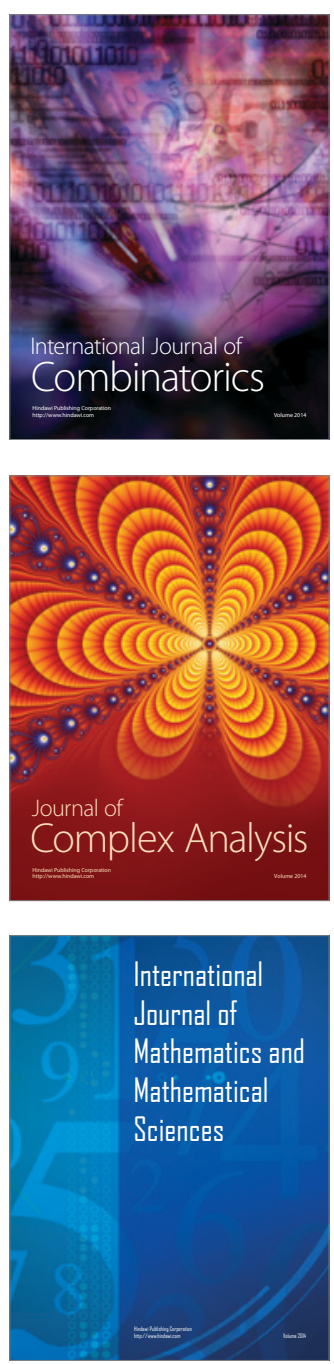
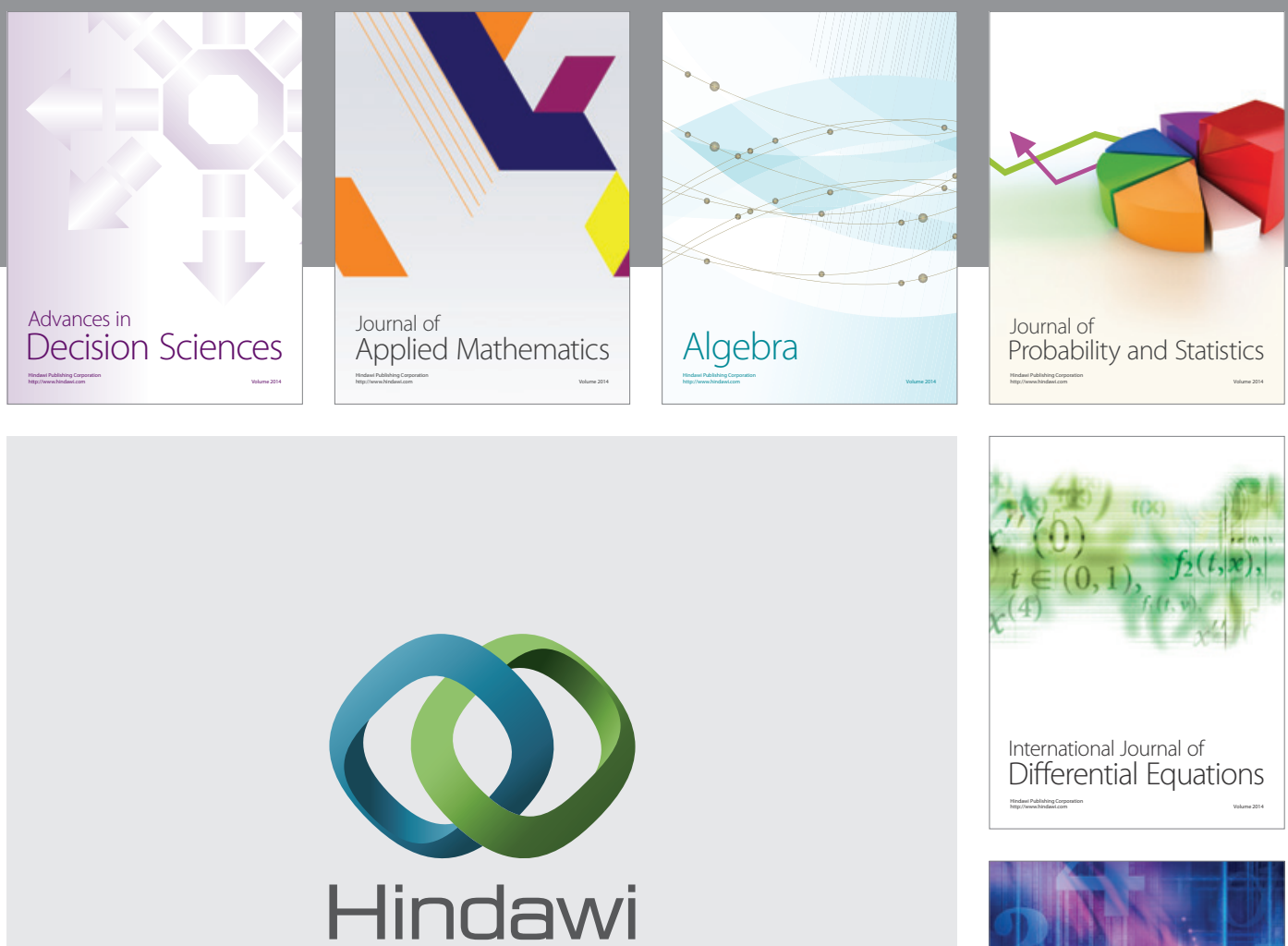

Submit your manuscripts at http://www.hindawi.com
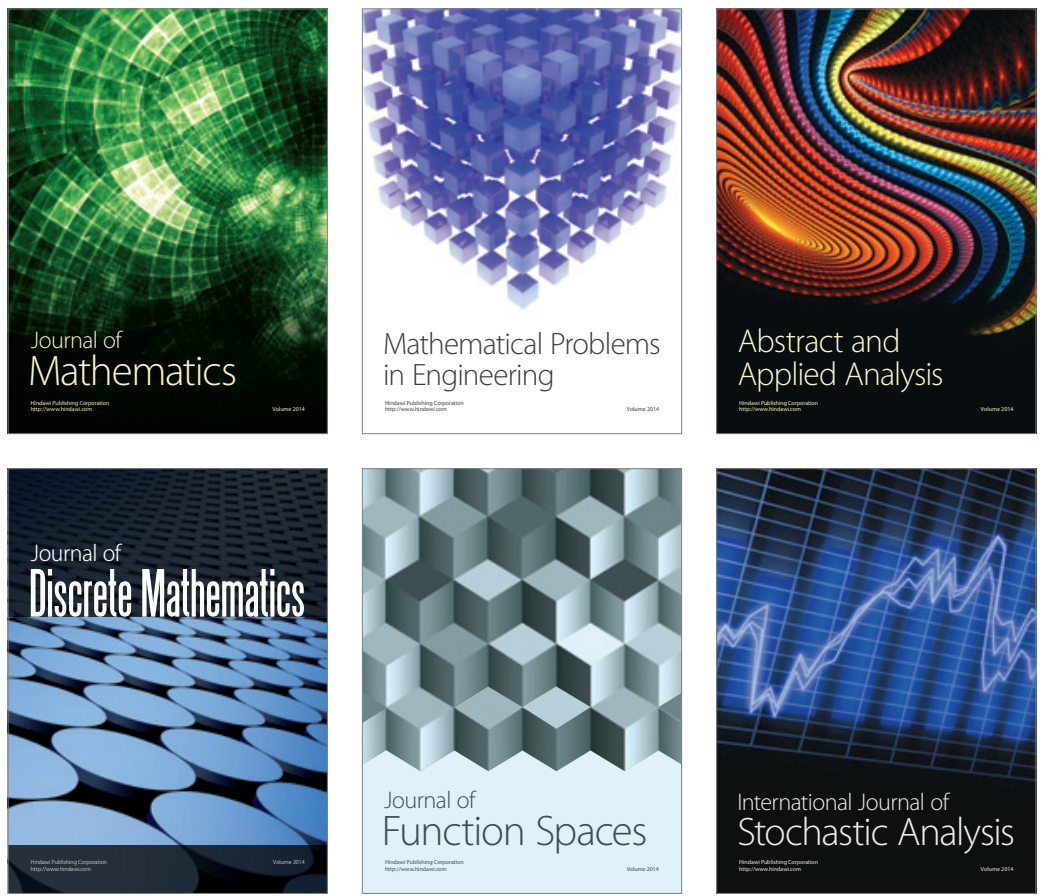

Journal of

Function Spaces

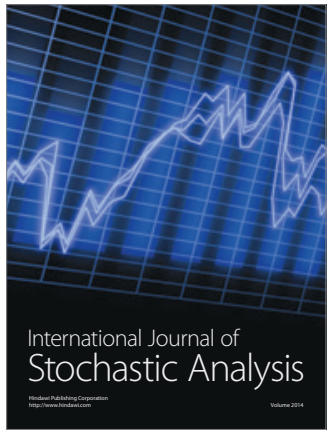

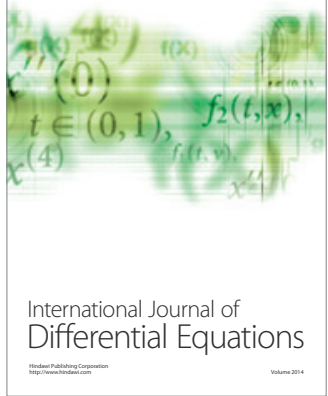
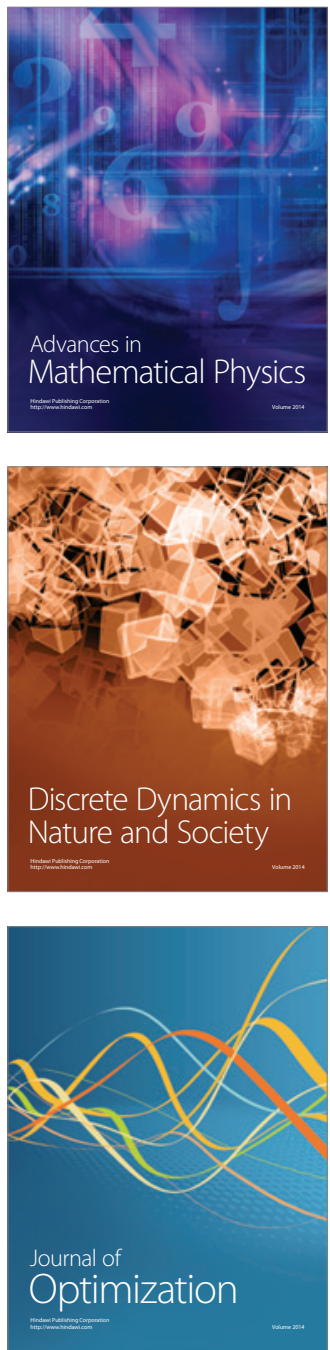